\title{
Endogenous endophthalmitis: a not so rare ophthalmic emergency among hemodialysis patients
}

\author{
Andreia Dias da Silva, Luísa Costa, Ana Rodrigues, Carolina Ferreira, Rosa Miguel, Marta Costa, Miguel Oliveira, Tiago Barra, Cátia Pêgo, \\ Giovanni Sorbo, Carla Lima, Jesús Garrido, Tânia Sousa, Edgar Gomes, Sérgio Lemos
}

Department of Nephrology of Centro Hospitalar Tondela-Viseu, Portugal

\section{ABSTRACT}

Endogenous endophthalmitis is an intraocular infection secondary to the hematogenous spread of microorganisms. It is an ophthalmological emergency associated with serious complications if not promptly diagnosed and treated. Despite the susceptibility of hemodialysis patients to episodes of bacteremia associated with vascular access, it is considered a rare disease. In fact, very few cases are described worldwide. We present 3 cases of endogenous endophthalmitis, secondary to bacteremia associated to vascular access in patients under hemodialysis treatment, aiming to raise awareness of this not so rare metastatic infection, possibly underdiagnosed in this population.

Keywords: Bacteremia; Endogenous endophthalmitis; Hemodialysis, Metastatic infection; Vascular access.

\section{INTRODUCTION}

Infectious endophthalmitis can be classified as exogenous or endogenous and is a medical emergency. Endogenous endophthalmitis (EE) is an intraocular infection associated to progressive inflammation of the vitreous and its adjacent structures, which occurs after the hematogenous spread of bacterial or fungal microorganisms. It is considered a rare disease that accounts for $2-8 \%$ of all cases of endophthalmitis ${ }^{1}$. The main risk factors for the development of EE include immunocompromised states (diabetes mellitus, end-stage renal and liver diseases, malignancy, chronic immunosuppressive therapy) underlying infections (mostly endocarditis, urinary tract infections, meningitis, liver abscesses), intravenous drug use, dental procedures and indwelling catheters. About half of reported EE cases are caused by bacteria and the other half by fungi ${ }^{2,3}$. In Europe, the most frequently identified causative bacteria are Staphylococcus aureus and Streptococcus pneumoniae ${ }^{1}$. Among fungal etiologies, Candida albicans is the most common yeast, and Aspergillus the most common mold ${ }^{4}$. Patients suffering from $\mathrm{EE}$ complain initially of progressive decreased vision and ocular pain. $\mathrm{EE}$ can present with or without signs and/or symptoms suggestive of bacteremia or fungemia. Frequent clinical findings include photophobia, ciliary and conjunctival injection, hypopyon, corneal edema, cells in the anterior chamber and vitreous cavity, and retinal and choroidal lesions. Bilateral ocular involvement can occur but, due to a more direct course through the right carotid artery, the right eye is infected more frequently.

The preferential vascular access for dialytic treatment in patients with stage 5 chronic kidney disease (CKD) on hemodialysis is the arteriovenous fistulae (AVF), which has less probability of infectious complications compared to central venous catheters (CVC) or even arteriovenous prosthesis. The relative risk of CVC-related bacteremia is estimated to be 10 times greater than the risk of bacteremia associated to $A V F^{5}$. Metastatic infections complicate catheter-related bacteremia in $5-10 \%$ of cases, with endocarditis being the most frequent. EE is described as a rare metastatic infection, with few cases reported worldwide ${ }^{7-9}$.

\section{CASE 1}

Female patient, 74 years old, with a history of type 2 diabetes and end-stage renal disease (ERSD) on hemodialysis through tunnelled CVC in the right internal jugular vein, presented with fever of 48 hours duration, arterial hypotension and purulent exudate from the CVC exit site. As a catheter related-bacteremia was suspected, the CVC was removed and samples of blood, as well as the CVC tip, collected for culture. The patient was hospitalized and initiated empiric antibiotic therapy with vancomycin and gentamycin. The cultures were positive for methicillinresistant Staphylococcus aureus, and vancomycin was maintained. On the fifth day of hospitalization, the patient presented symptoms of right ocular pain and blurred vision with reduced acuity. She was evaluated by Ophthalmology, who verified the presence of right conjunctival infection and hypopyon and made the diagnosis of EE. A single dose of intravitreal vancomycin and systemic corticotherapy was prescribed. At the time of discharge (day 45), the patient had reduction of visual acuity to the perception of luminosity and had suffered a retinal detachment. In follow-up, there was no improvement of visual acuity.

\section{CASE 2}

Female patient, 63 years old, with ERSD on hemodialysis through tunnelled CVC in the right internal jugular vein, type 2 diabetes and 
previous episodes of dialysis catheter-related bacteremia (2 of which complicated by metastatic infections - endocarditis and spondylodiscitis), presented intradialytic shivering with fever (auricular temperature of $39.5^{\circ} \mathrm{C}$ ), nausea and vomiting. After exclusion of other infectious foci, she was hospitalized with a diagnosis of catheter related-bacteremia. The CVC was removed and samples of blood, as well as the CVC tip, sent for culture. The patient initiated broad-spectrum empiric antibiotic therapy (vancomycin and gentamycin). The cultures were positive for methicillin-sensitive Staphylococcus aureus, and vancomycin was maintained. On the fourth day of hospitalization, the patient developed a sudden loss of vision in the right eye. After Ophthalmology's observation, the patient was submitted to an intravitreal vancomycin administration for an $\mathrm{EE}$. At the time of discharge (day 17) and in follow-up, the patient maintained reduced visual acuity.

\section{CASE 3}

Female patient, 55 years old, admitted to the emergency department for prostration, fever, ocular pain and left conjunctival hyperemia. She had a history of ESRD on hemodialysis through native arteriovenous fistula (area puncture) type 2 diabetes and arterial hypertension. Physical examination revealed prostration, fever (auricular temperature of $40{ }^{\circ} \mathrm{C}$ ), and arterial hypotension. There were no signs of eye trauma or inflammatory signs localized to the fistula. Blood analysis showed elevation of the inflammatory parameters (C-reactive protein $35 \mathrm{mg} / \mathrm{dl}[<0.5 \mathrm{mg} / \mathrm{dl}]$, procalcitonin $32.23 \mathrm{ng} / \mathrm{ml}$ [0.01-0.64 ng/ml]). Initial exams showed no evident infectious focus that explained the patient's septic status (even central nervous system infection and infective endocarditis were excluded). Patient was observed by Ophthalmology, with initial diagnosis of bacterial conjunctivitis. Blood samples were collected and empirical antibiotherapy started with vancomycin and meropenem. On third day of hospitalization, although afebrile, hemodynamically stable and with 15 points on the Glasgow scale, the left eye pain worsened, and the patient became amaurotic. Ophthalmology re-evaluation was promptly requested, and the diagnosis of EE in a probable context of septic embolization (secondary to inoculation of skin microorganisms during punctures of the arteriovenous fistula) was made. Methicillin-sensitive Staphylococcus aureus was isolated from blood cultures. Systemic corticosteroids and intravitreal vancomycin were added to the antibiotic therapy already started. However, on seventh day, the patient presented atalamia, pupillary hypopyon, conjunctival chemosis, stony consistency at digitopression, ophthalmoplegia, proptosis and no light perception in the left eye, and was therefore submitted to enucleation of the left eye.
Table 1 summarizes the clinical features and outcomes of the 3 cases reported.

\section{DISCUSSION AND CONCLUSIONS}

$E E$ is a metastatic infection less frequent than infectious endocarditis or spondylodiscitis in stage 5 CKD patients under a chronic hemodialysis program but should not be considered as a rare condition. Its indolent onset and clinician's (and even patient's) inattention/nonvalorization of usually mild ocular symptoms and signs at the early stage of the disease in septic scenarios may lead to late diagnosis and, unfortunately, to irreversible ophthalmologic sequelae. The 3 cases described occurred in a 6-year period in a single center and, given the diagnostic delay in all cases, there were permanent ocular sequelae, in one of them requiring enucleation of the affected eye.

In the presence of signs and symptoms suggestive of $E E$ in a patient undergoing hemodialysis, with or without evidence of a vascular access-related infection/bacteremia, urgent observation by Ophthalmology should be required. The diagnosis of EE is essentially based on clinical findings on ocular examination and easily corroborated by the presence of systemic signs and symptoms suggestive of bacteremia. A sample of vitreous fluid should ideally be obtained for culture (by vitreous tap using a needle and syringe or by vitrectomy, if indicated), in addiction to blood cultures (since they are only positive in one third of EE cases ${ }^{10}$ ). In the 3 cases described, the blood cultures identified the causative microorganism and when the diagnosis of EE was considered, the result was already available (dispensing with the need to collect vitreous fluid).

The treatment should be promptly initiated if there is suspicion of $E E$ and is based on the administration of intravitreal antibiotherapy, along with systemic antibiotics (with wide spectrum and good intraocular penetration). Since Gram-positive organisms are responsible for most dialysis vascular access-related infections (coagulase-negative Staphylococcal and S. aureus together account for 40 to $80 \%{ }^{11}$ ), the initial empiric systemic antibiotherapy in these patients generally includes vancomycin, and cover for Gramnegative (including Pseudomonas aeruginosa) with gentamycin or ceftazidime. Unfortunately, these 3 antibiotics have an intraocular penetrance that is generally poor and varies according to the pathogen in question ${ }^{12}$; therefore, early identification of EE and addition of intravitreal antibiotics will permit better outcomes. If a fungal infection is suspected, intravenous fluconazole or amphotericin-B should be administered.

Table 1

Clinical characteristics, etiological microorganisms and outcomes of the 3 cases reported.

\begin{tabular}{c|c|c|c|c||} 
Cases & Age & Comorbidity & Vascular access & Outcome \\
\hline 1 & 74 & Diabetes & Tunnelized CVC & Methicillin-resistant Staphylococcus aureus \\
2 & 63 & Diabetes & Tunnelized CVC & Methicillin-sensitive Staphylococcus aureus \\
3 & 55 & Diabetes & AVF & Methicillin-sensitive Staphylococcus aureus
\end{tabular}

CVC - Central venous catheter, AVF - arterial venous fistulae. 
The preferred intravitreal antibiotics for bacterial EE are ceftazidime $(2.25 \mathrm{mg} / 0.1 \mathrm{ml})$ and vancomycin $(1 \mathrm{mg} / 0.1 \mathrm{ml})$, although amikacin or clindamycin can be used, for penicillin-allergic patients. In case of fungal $\mathrm{EE}$, intravitreal amphotericin-B $(5-10 \mu \mathrm{g} / 0.1 \mathrm{~mL})$ or, less frequently, voriconazole $(100 \mu \mathrm{g} / 0.1 \mathrm{~mL})$ are options ${ }^{12}$. Patients are monitored daily for response to treatment and if, after 3 days, there is worsening inflammation, declining vision, or increasing pain, the intravitreal antibiotic can be repeated, a pars plana vitrectomy can be done, although it is not a widely accepted procedure ${ }^{10,12}$. The concomitant use of systemic corticotherapy is not also widely accepted, but it is used regularly to better infection control. Currently, there's no evidence to support the routine use of intravitreal corticotherapy ${ }^{13}$.

EE is a serious, and probably underdiagnosed, condition that can affect hemodialysis patients, and we should be alert to its occurrence among this susceptible population, giving the poor prognosis if it is not promptly identified.

Disclosure of potential conflicts of interest: none declared.

\section{References}

1. Jackson TL, Paraskevopoulos T, Georgalas I. Systematic review of 342 cases of endogenous bacterial endophthalmitis. Surv Ophthalmol. 2014; 59(6):627-35

2. Binder MI, Chua J, Kaiser PK, Procop GW, Isada CM. Endogenous endophthalmitis: an 18-year review of culture-positive cases at a tertiary care center. Medicine (Baltimore). 2003; 82(2):97-105

3. Connell PP, O'Neill EC, Fabinyi D, et al. Endogenous endophthalmitis: 10-year experience at a tertiary referral centre. Eye. 2011; 25(1):66-72

4. Lingappan A, Wykoff CC, Albini TA, et al. Endogenous fungal endophthalmitis: causative organisms, management strategies, and visual acuity outcomes. Am J Ophthalmol. 2012; 153(1):162-66

5. Taylor $\mathrm{G}$, Gravel D, Johnston L, et al. Incidence of bloodstream infection in multicenter inception cohorts of hemodialysis patients. Am J Infect Control. 2004; 32:155

6. Allon M. Dialysis catheter-related bacteremia: treatment and prophylaxis. Am J Kidney Dis 2004; 44:779

7. Kuo G, Lu YA, Sun WC, et al. Epidemiology and outcomes of Endophthalmitis in chronic dialysis patients: a 13-year experience in a tertiary referral center in Taiwan. BMC Nephrol. 2017; 18(1):270

8. Saleem MR, Mustafa S, Drew PJ, et al. Endophthalmitis, a rare metastatic bacterial complication of haemodialysis catheter-related sepsis. Nephrol Dial Transplant 2007; 22:939-41

9. Cabana-Carcasi ML, Becerra-Mosquera V, González-Tabarés L, Novoa-García D. Endogenous endophthalmitis as a complication of sepsis related to a tunnelled haemodialysis catheter. Nefrologia. 2012; 32(2):255-6

10. Vaziri K, Schwartz SG, Kishor K, Flynn HW. Endophthalmitis: state of the art. Clin Ophthalmol. 2015; 9:95-108

11. Fysaraki M, Samonis $G$, Valachis $A$, et al. Incidence, clinical, microbiological features and outcome of bloodstream infections in patients undergoing hemodialysis. Int J Med Sci. 2013; 10(12): 1632-8

12. Birnbaum F, Gupta G. Endogenous Endophthalmitis: diagnosis and treatment. Eyenet Magazine. 2016; 33-5

13. Bui DK, Carvounis PE. Evidence for and against intravitreous corticosteroids in addition to intravitreous antibiotics for acute endophthalmitis. Int Ophthalmol Clin. 2014; 54(2): 215-24

\section{Correspondence to:}

Andreia Dias da Silva, MD

Department of Nephrology of Centro Hospitalar Tondela-Viseu, Portugal

E-mail: andreiaraquelsilva89@gmail.com 